\title{
Differential response gradients to a Pavlovian safety signal following active avoidance training in young and adult rats
}

\author{
JAMES F. BRENNAN \\ State University College at Buffalo, Buffalo, New York 14222
}

\begin{abstract}
Following 300 training trials in two-way shuttle avoidance signaled by a tone (CS+), two groups each of weanling and adult rats were given Pavlovian discrimination training in which the CS+ was followed by inescapable shock, and a more intense tone (CS-) signaled no shock. An additional group at each age level received both tones paired randomly with shock or no shock. Subsequent generalization tests along the frequency dimension indicated that both pups and adults tested at the CS+ intensity showed similar gradients of frequency control. Gradients for the adults tested at the CS- intensity tended to be inverted, with least responding at $\mathrm{CS}-$, a result not found in the young subjects. The results were considered in light of Pavlovian extradimensional influences on the control of avoidance behavior.
\end{abstract}

Studies of the developmental aspects of aversively motivated behavior have typically reported a deficit in young rats when the dependent variable involved a passive avoidance response inhibition (e.g., Schulenburg, Riccio, \& Stikes, 1971). In contrast, little systematic difference across ages has been found in the acquisition of active avoidance responding (e.g., Kirby, 1963; Riccio, Rohrbaugh, \& Hodges, 1968), and similar generalization gradients of active avoidance behavior have been demonstrated in young and adult rats (cf. Brennan \& Riccio, 1972b; Rohrbaugh, Brennan, \& Riccio, 1971). However, in a study by Brennan and Riccio (1972c), age differences in the control of active avoidance were obtained after interpolation of intradimensional Pavlovian discrimination training between acquisition of the instrumental response and generalization tests along the CS tonal frequency dimension. Brennan and Riccio suggested that the disparity in gradients may reflect a deficit in the response inhibitory component, presumably associated with the safety signal during discrimination training, on the part of immature subjects.

Desiderato (1969) initially trained dogs in Sidman avoidance and separately paired tone and click stimuli with either the presence (CS+) or absence (CS-) of shock. Subsequent tests of generalization effects upon the avoidance baseline indicated that relative gradients of inhibition and excitation were comparable. Another

Portions of this experiment were presented at the meeting of the Eastern Psychological Association in Philadelphia, April, 1974. This research was supported by Grant No. 021-7151-A from the Research Foundation of State University of New York and Grant No. 21-9303-F from State University College at Buffalo. The manuscript was prepared while the author was in residence at the Nencki Institute of Experimental Biology, Warsaw, supported by a research fellowship from the International Research Exchanges Board, and the assistance of the institute's technical staff is appreciated. Requests for reprints should be sent to the author, Department of Psychology, State University College, 1300 Elmwood Avenue, Buffalo, New York 14222 . series of experiments that demonstrated the effectiveness of Pavlovian procedures to generate gradients of inhibitory control were reported by Moore (1972), who investigated the effects of various Pavlovian discriminative manipulations on the rabbit's nictitating membrane response. Of interest was his finding that subjects given tonal CS intensity discrimination training and subsequently tested for frequency generalization at the intensity value of $\mathrm{CS}-$, a safety signal, yielded characteristic inhibitory gradients. It seemed that the procedure described by Moore might be of use to examine response inhibition of active avoidance behavior in young rats. That is, response speed to fear and safety signals offered an alternative index to passive avoidance assessments of behavioral differences in inhibition due to age.

\section{METHOD}

\section{Subjects}

The young subjects were 18 albino Sprague-Dawley rats, purchased from Carsworth Company, that were 22 days of age when training began (weaning occurred at 20 days). The remaining 18 subjects were experimentally naive male rats of approximately 120 days of age. The pups were housed in pairs and the adults singly with food and water available ad lib.

\section{Apparatus}

A twu-compartment chamber made of clear acrylic plastic was used for avoidance training. Each compartment measured $30 \times 22 \times 37 \mathrm{~cm}$, and a center partition with a 9-cm-square opening separated both compartments. Footshock from a matched impedance source (Campbell \& Teghtsoonian, 1958) was delivered through a Foringer scrambler to the chamber floor, which consisted of $.2-\mathrm{cm}$ steel rods, spaced $1 \mathrm{~cm}$ apart. Photoelectric cells were located $15 \mathrm{~cm}$ from the door opening in each compartment. For the pups, acrylic inserts, measuring $30 \times 14 \times 25 \mathrm{~cm}$, were used to decrease the overall area in each compartment, although the distance between photocells was not affected. A Heathkit audio generator provided tonal frequency values of $2,000,2,500,3,000,3,500$, and $4,000 \mathrm{~Hz}$ and two 


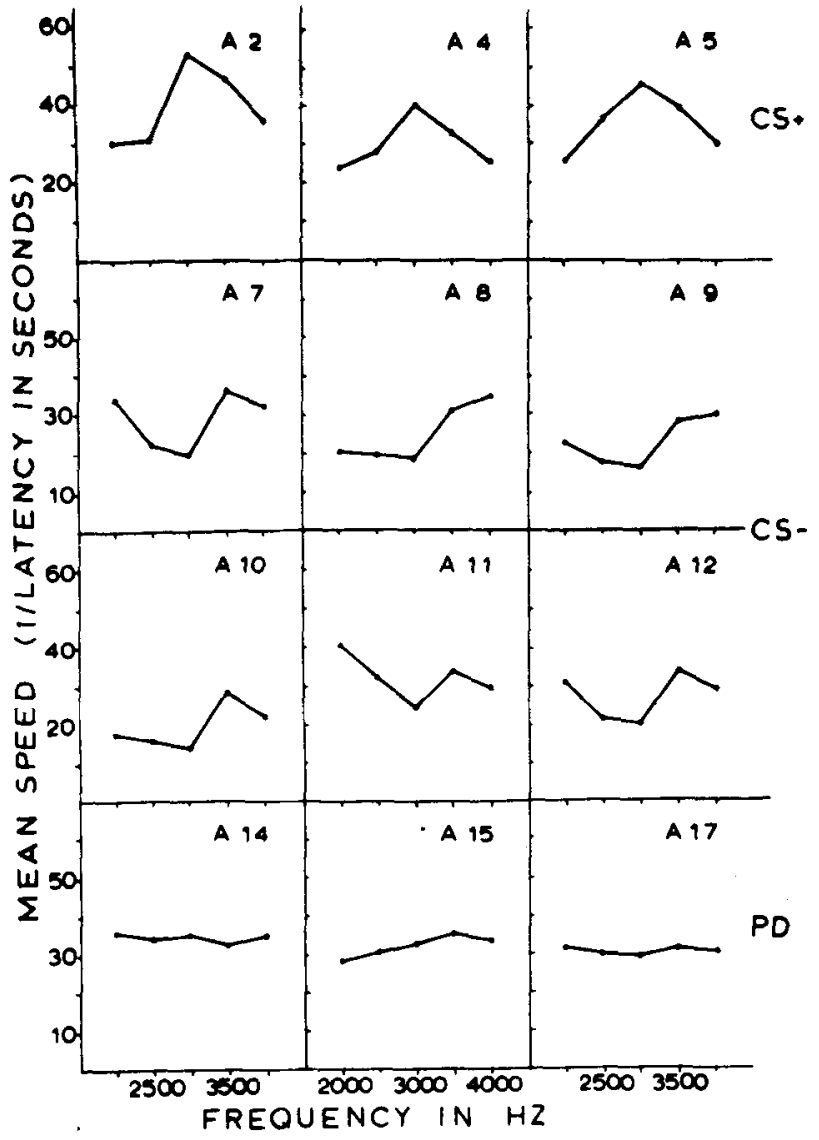

Figure 1. Individual frequency gradients from three representative adults when tests were conducted at the CS+ intensity (upper), and gradients from all subjects tested at the CS- intensity value. The bottom panel contains frequency gradients from three subjects after pseudodiscrimination training along CS intensity.

gradations of intensity used in this experiment, which were measured at 66 and $77 \mathrm{~dB}\left(.0002 \mathrm{dyne} / \mathrm{cm}^{2}\right)$ by a sound level meter (General Radio Corp., Type 1551-C) at the 3,000-Hz frequency in the center of the chamber. Tonal stimuli were presented through two 6-in. speakers mounted on the acrylic lids covering each compartment. White noise delivered to the experimental room attenuated extraneous sounds, with the ambient noise level measured at $63 \mathrm{~dB}$ SPL in the center of the chamber. A Standard Electric timer provided measurement of response latency to .01 of a second. The only source of illumination was provided by a $15-\mathrm{W}$ bulb suspended over the center of the chamber. For the Pavlovian conditioning phase of the experiment, all subjects were given tone and shock presentations in an acrylic chamber measuring $22 \mathrm{~cm}$ square, with a grid floor. The sources of tones and shock were the same as described for the avoidance chamber.

\section{Procedure}

The overall plan involved individually training and testing the subjects under three phases of experimentation:

Avoidance training. Each subject was trained in two-way active shuttle avoidance for 100 trials on 3 successive days, making a total of 300 training trials. Prior to the 1 st day's training, subjects were permitted five spontaneous cross-through responses. On a given training trial, the $3,000-\mathrm{Hz} 66-\mathrm{dB} \mathrm{CS}$ was followed $5 \mathrm{sec}$ later by the $175-\mathrm{V}$ shock US, unless a shuttle response was emitted, which precluded US onset and terminated the tone. Responses made after the 5 -sec ISI coterminaied both tone and shock. Trials were separated by a variable interval averaging $30 \mathrm{sec}$. Intertrial crossings did not affect the avoidance contingency, since interruption of a photocell beam opened that circuit until the beam in the opposite compartment had been broken. Three adults and two pups were discarded from the experiment for failing to emit at least 25 avoidance responses by Day 2 of training. These subjects were replaced with naive rats.

Pavlovian intensity discrimination training. On the day following the third avoidance training session, subjects at each age level were assigned to one of three discriminative treatment conditions. For two groups of adults and two groups of pups, subjects were placed in the small chamber and presented with 50 pairings of the original avoidance training intensity value of $66 \mathrm{~dB}(\mathrm{CS}+)$ followed $5 \mathrm{sec}$ later by inescapable shock of $.5 \mathrm{sec}$ duration and 50 presentations of a more intense tone of $77 \mathrm{~dB}$ (CS-), which was never paired with shock and terminated after $5 \mathrm{sec}$. The remaining group at each age level served under a control condition by receiving both intensity values with shock randomly paired an equal number of times with each stimulus. For all six groups, the frequency value for both intensity stimuli was held constant at $3,000 \mathrm{~Hz}$. Stimulus presentations were separated by a variable interval averaging $40 \mathrm{sec}$, and the order of stimulus events was random with the single restriction that the same event did not occur more than three times consecutively.

Frequency generalization testing. Approximately $4 \mathrm{~h}$ after the Pavlovian treatment, each subject was retumed to the shuttle chamber, and acquisition training was reinstated until a criterion of five consecutive avoidance responses was recorded. The shock was then disconnected and a frequency generalization test proceeded in avoidance extinction. Tones of $2,000,2,500$, $3,000,3,500$, and $4,000 \mathrm{~Hz}$ were presented randomly within blocks of five test trials. Of the four intensity true-discrimination training conditions, one group each of adults and pups were tested for frequency generalization at the CS+ intensity, while the other two groups at each age level were tested at the louder intensity value (CS-). The young and adult subjects that received random shock and tonal intensity presentations were given the frequency generalization test at the more intense value. If a subject failed to respond on a test trial, the tone automatically terminated after the elapse of $12 \mathrm{sec}$, and the testing session was ended if the subject failed to respond for five consecutive trials.

\section{RESULTS}

Response latencies to each generalization test value were converted to speed scores by calculating their reciprocals. In the top panel of Figure 1, gradients from three representative subjects in the adult group tested for frequency generalization at the CS+ intensity value are presented. Reasonably symmetrical decrements in response speed around the CS+ tonal stimulus were obtained from the subjects in this group. The individual gradients from the six subjects tested at the CSintensity are shown in the middle panels of Figure 1. While the gradient slopes were not as sharp as in the CSt group, response speeds to the $3,000-\mathrm{Hz}$ test value were less than the other test stimuli in all subjects. The bottom panel of Figure 1 contains the results after pseudodiscrimination intensity training from three representative subjects, which indicate complete frequency generalization to all test values. Comparable data from the pups are presented in Figure 2. The gradients from three of the young subjects tested at the 
CSt intensity indicate overall decreasing vigor of response speed around the $3,000-\mathrm{Hz}$ value. The individual gradients from the six pups tested at the CSintensity, contained in the middle panels of Figure 2, reflect little effect of frequency, with two subjects ( $P 9$ and $P$ 12) responding least to test values other than $3,000 \mathrm{~Hz}$. Similarly, the flat gradients from three representative pseudodiscriminative control pups, in the bottom panel of Figure 2, do not show any systematic frequency effect. Overall analysis of these data revealed significant effects from age $(F=5.79, \mathrm{df}=1 / 30$, $\mathrm{p}<.05)$, discrimination treatment $(\mathrm{F}=3.48, \mathrm{df}=2 / 30$, $\mathrm{p}<.05$ ), and the Age by Treatment interaction $(F=3.34, d f=2 / 30, p<.05)$. In addition, the effect from stimuli used in testing $(F=2.5, d f=4 / 120$, $\mathrm{p}<.05$ ) and the interaction of Stimuli by Treatment $(F=7.51, \mathrm{df}=8 / 120, \mathrm{p}<.001)$ were significant sources of variability, while the remaining interactions of Age by Stimuli and Age by Treatment by Stimuli were insignificant (Fs $<1)$. The level of responding, collapsed across stimuli, was significantly less (Newman-Keuls, $\mathrm{p}<.05)$ in the adult CS-group $(\overline{\mathrm{X}}=.26)$, than in the other adult groups $(\overline{\mathrm{X}} \mathrm{s}=.33, \mathrm{CS}+; .35, \mathrm{PD})$. However, a similar comparison among the young subjects revealed that the pups tested at the CS+ value $(\overline{\mathrm{X}}=.34)$ differed in overall responding from the remaining pup groups $(\overline{\mathrm{X}} \mathrm{s}=.22$, CS $-; .21, \mathrm{PD})$. To examine further the relationship between age and the true-discriminative treatments, separate analyses were done between ages from the CSt and CS- groups. While the effect of generalization stimuli was highly significant under the CS+ testing condition $(F=32.57, \mathrm{df}=4 / 40, \mathrm{p}<.001)$, neither the main effect of age nor the Age by Stimuli interaction $(\mathrm{F}<1)$ were significant. Comparison between age groups tested at the CS- intensity did not indicate a systematic effect from age, while the effect of test stimuli only approached the acceptable significance level $(F=2.56$, df $=4 / 40, p<.10)$. However, the Age by Stimuli interaction attained significance for the CSgroups $(F=10.57, \mathrm{df}=4 / 40, \mathrm{p}<.001)$.

Among other measures, analysis of the number of generalization test trials indicated the presence of an age effect $(F=8.49$, df $=1 / 30, p<.01)$, with the young subjects extinguishing more rapidly, a finding consistent with previous evidence of age differences in resistance to extinction (e.g., Egger \& Livesey, 1972; Wilson, Phinney, $\&$ Brennan, 1974). The significant main effect of discrimination treatment $(\mathrm{F}=41.1, \mathrm{df}=2 / 30, \mathrm{p}<.001)$ supported the finding that, within each age level, the CS+ groups responded for the longest duration, while the CS- subjects extinguished most rapidly. That resistance to extinction was not differentially affected by age with each treatment was confirmed by the lack of an interaction $(F<1)$ between these variables.

As a measure of overall activity during generalization testing, the number of intertrial crossings for each subject was recorded. Analysis of the ratio of crossings

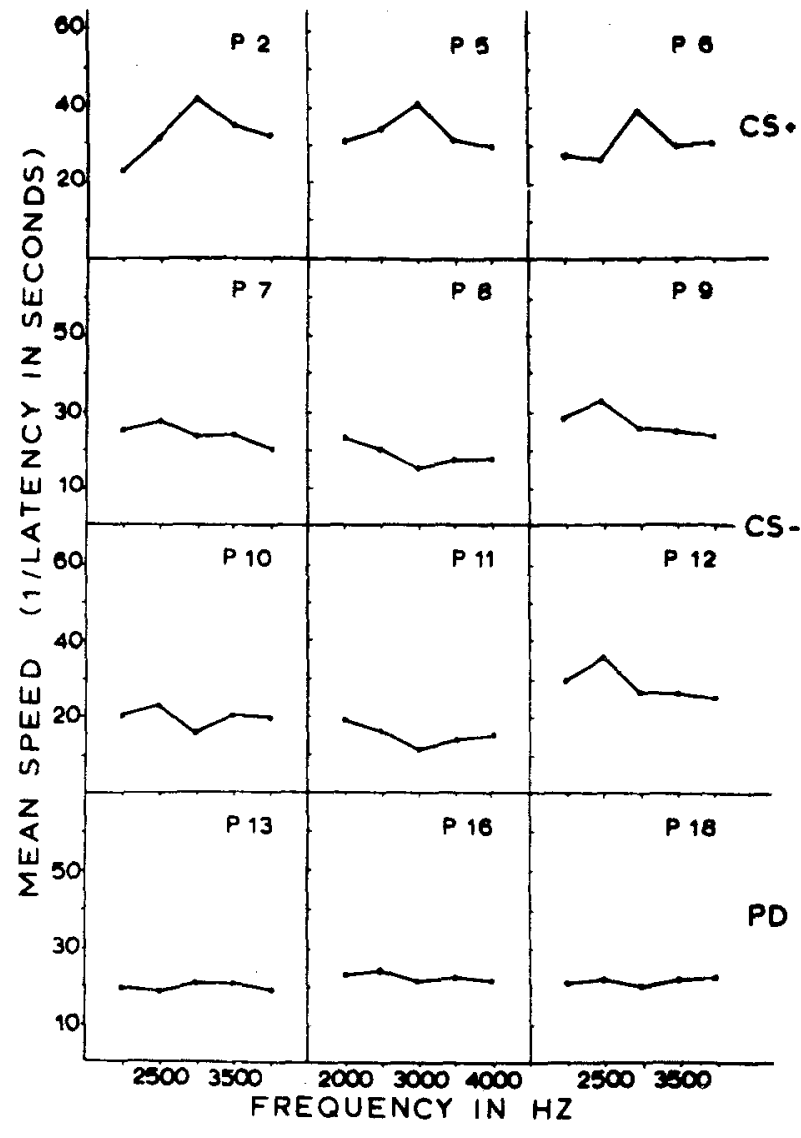

Figure 2. Individual frequency gradients from the pups: The upper panel shows results from three representative subjects tested at the CS+ intensity, the middle panels contain the gradients from subjects tested at the CS- intensity, and the bottom panel indicates three representative gradients obtained after intensity pseudotraining.

to the total number of test trials indicated greater activity in the pups than their adult counterparts $(F=21.87, d f=1 / 30, p<.001)$. Further, within the pup groups, activity under the CS- and PD conditions was greater than the CS+ treatment, while the former groups did not differ from each other.

\section{DISCUSSION}

Among the three adult groups, differences in stimulus generalization on a tonal frequency dimension were found under the training and testing conditions. The amount of frequency control reflected in the adult gradients obtained at the CS+ fear intensity value was consistent with data showing tonal dimensional control after extended discrete trial avoidance training (Rohrbaugh et al., 1971). Similarly, the data are in agreement with frequency generalization gradients, as reported by Brennan and Riccio (1973), obtained after Pavlovian extradimensional intensity discrimination training.

The frequency gradients from the adults tested at the 
CS - safety intensity showed an overall depression, with slowest responding at the $3,000-\mathrm{Hz}$ value. In their study, Brennan and Riccio (1973) reported the effectiveness of various discriminative procedures upon CS frequency and intensity, which suggested a relative independence between the tonal dimensions. That is, discrimination training affected the symmetry of the decremental slope around the original CS frequency, whereas variation in the CS intensity dimension consistently yielded a dynamism effect that was modified, but not eliminated, by discrimination training (see also Brennan \& Riccio, 1972a; Zielinski, 1965). To avoid confounding the present findings by the persistence of auditory dynamism, the safety value (CS-) was designated as a more intense stimulus than the fear signal (CSt). Nevertheless, the lack of sharpness in the gradients from the adult CS- group (cf. Moore, 1972), relative to the excitatory gradients of the CS+ group, presumably reflects a generalized influence from the $3,000-\mathrm{Hz}$ tone of the less intense value that had been experienced by subjects as both the avoidance CS and the CS+ used during discrimination training. It is also interesting to note that Desiderato (1970) found little evidence showing inhibition of Sidman avoidance in dogs when both CS+ and CS- were tones, a finding that tends to support the present indication of excitatory generalization between stimuli. Accordingly, while the frequency and intensity dimensions may be independently sensitive to discriminative procedures and yield differently shaped generalization gradients, the present findings indicate that the dimensions are not completely orthogonal.

The pseudodiscriminative control condition in the present study involved a type of noncontingent, extradimensional influence from intensity upon frequency generalization. A similar procedure, used by Brennan and Riccio (1973), resulted in a decremental effect upon frequency control when the frequency generalization test was administered at the intensity of the original avoidance CS. That frequency control was eliminated in the PD adults of the present study suggests a more pronounced extradimensional effect when the test values are presented at the more intense level of the stimulus used in the pseudotraining procedure but not employed as the warning signal during avoidance training. Although the adult frequency gradients in the PD condition were relatively flat, an overall high level of responding across test stimuli was retained. It is unclear whether the elevated gradients represent the retention of learned fear to all frequency values due to noncontingent pairings of the two intensities with shock or whether they are the result of a performance artifact from the synergistic effect involved in testing frequency at the more intense value.

The frequency gradients obtained from the young subjects tested at the CS+ fear intensity value are consistent with expectations based upon previous findings. Specifically, the marked similarity in frequency control between ages is in agreement with data obtained after discrete trial avoidance training (Brennan \& Riccio, 1972b). The pup gradients suggest further that extradimensional true-discrimination training along CS intensity results in comparable frequency control between ages when testing is at the value of CSt.

The lack of any clear tendency toward reliable inhibitory gradients along frequency in the pups tested at the $\mathrm{CS}$ - intensity value indicates that true-extradimensional influences were minimal at the safety intensity. When the flat and relatively depressed gradients of the pups tested after pseudodiscrimination training are also considered, the data from both young groups suggest functionally equivalent effects. Accordingly, a deficit in inhibitory control of active avoidance behavior in the pups was found after Pavlovian discriminative procedures. It may be that testing the pups at the more intense value under both the CS- and PD conditions served a discriminative function by signaling a change in the aversive context of the testing situation. The increase of intertrial activity in the young CS - and PD groups offers indirect support for the suggestion of an acquired deficit in attention to the louder tones. Accordingly, in contrast to their adult counterparts, the pups tested at the louder intensity value may have perceived all testing tones as irrelevant and began to engage in other (e.g., curiosity related) behavior between trials, producing flat gradients of overall slow speed.

\section{REFERENCES}

Brennan, J. F., \& Riccio, D. C. Persistence of dy namism effects in rats following auditory intensity differentiation. Leaming and Motivation, 1972, 3, 259-271. (a)

Brennan, J. F. \& Riccio, D. C. Stimulus generalization along dimensions of an active avoidance $\mathrm{CS}$ in young rats. Psychonomic Science, 1972, 29, 170-172. (b)

Brennan, J. F., \& Riccio, D. C. Stimulus control of shuttle avoidance in young and adult rats. Canadian Journal of Psy chology, 1972, 26, 361-373. (c)

Brennan, J. F., \& Riccio, D. C. Stimulus control of avoidance behavior in rats following differential or nondifferential Pavlovian training along dimensions of the conditioned stimulus. Journal of Comparative and Physiological Psy chology, 1973, 85, 313-323.

Campbell, B. A., \& Teghtsoonian, R. Electrical and behavioral effects of different types of shock stimuli on the rat. Journal of Comparative and Physiological Psychology, 1958,51, 185-192.

Desiderato, $O$. Generalization of excitation and inhibition in control of avoidance responding by Paviovian CSs in dogs. Joumal of Comparative and Physiological Psychology, 1969, 68, 611-616.

Desiderato, 0 . Transituational control of avoidance responding by Pavlovian CSs. Psy chonomic Science, 1970, 19, 11-13.

Egger, G. J., \& Livesey, P. J. Age effects in the acquisition and retention of active and passive avoidance learning by rats. Developmental Psy chobiology, 1972, 5, 340-351.

Kirby, R. H. Acquisition, extinction, and retention of an avoidance resoonse in rats as a function of age. Joumal of Comparative and Physological Psychology, 1963, 56, 158-162.

Moore, J. W. Stimulus control: Studies of auditory generalization in rabbits. In $A$. H. Black and W. F. Prokasy (Eds.), Classical conditioning II Current theory and research. New York: Appleton-Century-Crofts, 1972. 
Riccio, D. C. Rohrbaugh, M. \& Hodges, L. A. Developmental aspects of passive and active avoidance learning in rats. Developmental Psy chobiology, 1968, 1, 108-111.

Rohrbaugh, M., Brennan, J. F., \& Riccio, D. C. Control of two-way shuttle avoidance in rats by auditory frequency and intensities. Joumal of Comparative and Physological Psychology, 1971, 75, 324-330.

Schulenburg, C. J., Riccio, D. C., \& Stikes, E. R. Acquisition and retention of a passive-avoidance response as a function of age in rats. Joumal of Comparative and Phy siological Psychology, $1971,74,75-83$.
Wilson, L. M., Phinney, R. L., \& Brennan, J. F. Age-related differences in avoidance behavior in rats following $C S$ pre-exposure. Developmental Psychobiology, 1974, 7, $421-427$.

Zielinski, $K$. The influence of stimulus intensity on the efficacy of reinforcement in differential training. Acta Biologicae Experimentalis, 1965, 25, 317-335.

(Received for publication December 6, 1974; revision accepted February $4,1975$. ) 